\title{
VORTEX FORCE ANALYSIS OF WAVE-DRIVEN NEARSHORE CIRCULATION
}

Morteza Derakhti, Johns Hopkins University, derakhti@jhu.edu

Robert A. Dalrymple, Johns Hopkins University, rad@jhu.edu

It has been now well established that the alongshore variability of the incident wave heights and breaking crests have a profound effect in the wave-driven nearshore circulation. Wei et al. (2017) examined the free surface evolution and vertical vorticity generation by short-crested wave breaking in the surf zone. They showed that the normally-incident short-crested wave field is characterized by the formation of isolated breakers near the nodal lines, in addition to breaking at anti-nodal lines, leading to the bifurcation of breaking crests (Figure 1) and the generation of secondary circulation cells (Figure 2, arrows). However, the generation mechanism and the relative importance of the various wave-averaged forcing mechanisms of such complex circulation system remain unclear.

Useful formulations of wave-averaged forcing are based on either 1) the radiation stress concept (LonguetHiggins and Stewart, 1964), where forcing is written as the divergence of a stress tensor expressing excess wave momentum flux, or 2) on the vortex force formalism (Craik and Leibovich, 1976), where wave forcing is expressed through a combination of terms including vortex force and the dissipation of wave energy. Both formalisms have been successfully used to explain a range of hydrodynamic processes in the nearshore region where the latter has been drawing more and more attention during the recent years. The main advantage of the vortex force formalism is the clear separation of the conservative and nonconservative forcing mechanisms.

In this presentation, we examine 3-D structure of nearshore circulation driven by short-crested wave breaking using the 3-D Smooth Particle Hydrodynamic model, GPUSPH (Hérault et al., 2010). The alongshore variation of incident wave field has been imposed by using the method of intersecting wave trains proposed by Dalrymple (1975). We use the 3-D vortex force formalism to analyze the various forcing mechanisms of the observed circulation. Of particular interest is the relative importance of the vortex force compared with the other wave-averaged forces. The accuracy of the depth-averaged vortex force based on the formulation of Smith [2006] is also examined.

To investigate the wave forcing of the Eulerian current field, the phase-resolved 3-D Lagrangian results are first interpolated onto a Eulerian uniform grid and then are post-processed based on the vortex force formalism. The magnitude of the various forcing terms is examined across the domain revealing the relative contribution of each to the wave forcing. Figure 2 shows the dominant forcing mechanisms in the cross-shore and alongshore directions for the short-crested case shown in Figure 1. Further, the 3-D mean velocity field (not shown) reveals that the generated primary rip currents near the nodal lines have a considerable positive shear in and near the initial breaker zone. As the rips leave the surf zone, however, the velocity shear becomes weaker and more concentrated near the surface while the lower part of the velocity profile is approximately depth-uniform.

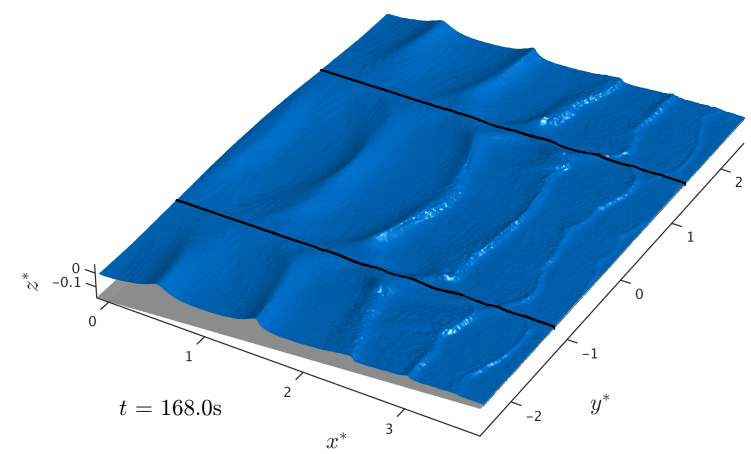

Figure 1 - Snapshots of the free surface elevations for a short-crested case. Black lines show the nodal lines.
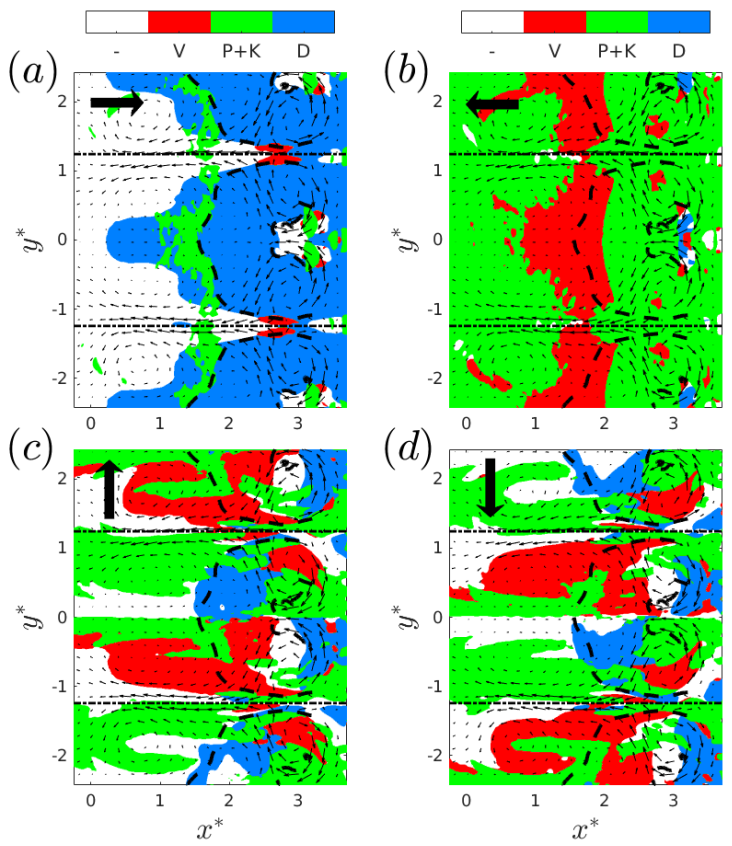

Figure 2 - Dominant wave-averaged forcing mechanisms in the (a) $+x$, (b) $-x,(c)+y$, and (d) $-y$ directions for the shortcrested case shown in Figure 1. Red, green and blue represent vortex force, the gradient of mean pressure and the Bernoulli head, and wave energy dissipation respectively. Arrows show the depth-averaged mean current. Dashed-lines show the initial breaking zone. Dotted-dashed lines show the nodal lines.

\section{REFERENCES}

Craik, Leibovich (1976), A rational model for Langmuir circulations, J. Fluid Mechanics, 73, 401-426.

Hérault, Bilotta, Dalrymple (2010), SPH on GPU with CUDA, J. Hydraulic Research, 48, 74-79.

Longuet-Higgins, Stewart (1964), Radiation stresses in water waves; physical discussion, with applications, in Deep Sea Res., vol. 11, pp. 529-562, Elsevier.

Smith (2006), Wave-current interactions in finite depth, J. Phys. Oceanogr., 36(7), 1403-1419.

Wei, Dalrymple, Xu, Garnier, Derakhti (2017), Shortcrested waves in the surf zone, J. Geophys. Res., 122, 4143-4162. 